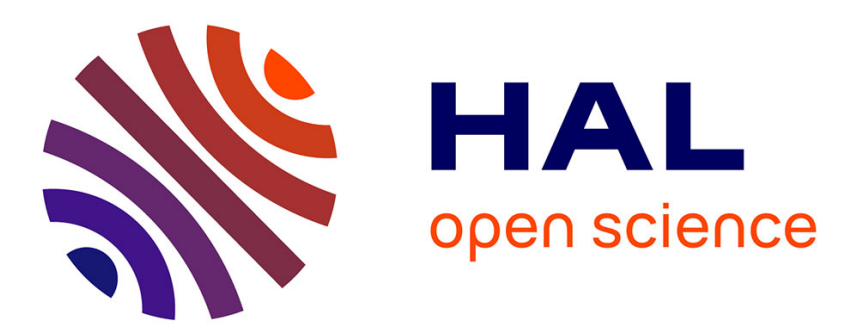

\title{
Evaluation of a multimodal urban arterial: the passenger macroscopic fundamental diagram
}

Nicolas Chiabaut

\section{To cite this version:}

Nicolas Chiabaut. Evaluation of a multimodal urban arterial: the passenger macroscopic fundamental diagram. Transportation Research Part B: Methodological, 2015, 81 (Part 2), 24 p. 10.1016/j.trb.2015.02.005 . hal-01215733v2

\section{HAL Id: hal-01215733 \\ https://hal.science/hal-01215733v2}

Submitted on 21 Apr 2017

HAL is a multi-disciplinary open access archive for the deposit and dissemination of scientific research documents, whether they are published or not. The documents may come from teaching and research institutions in France or abroad, or from public or private research centers.
L'archive ouverte pluridisciplinaire HAL, est destinée au dépôt et à la diffusion de documents scientifiques de niveau recherche, publiés ou non, émanant des établissements d'enseignement et de recherche français ou étrangers, des laboratoires publics ou privés. 


\section{Evaluation of a multimodal urban arterial:}

\section{2 the passenger Macroscopic Fundamental Diagram}

3

4

$5 \quad$ Nicolas Chiabaut*

6

$7 \quad *$ Corresponding author

8 Email: nicolas.chiabaut@entpe.fr

9 nicolaschiabaut.weebly.com

10

11 Université de Lyon

12 IFSTTAR / ENTPE

13 Laboratoire Ingénierie Circulation Transport LICIT

14 Rue Maurice Audin

15 F-69518 Vaulx-en-Velin, France 


\section{ABSTRACT}

21 This paper aims to extend the concept of Macroscopic Fundamental Diagram (MFD) to

22 combine different transportation modes. Especially, we propose a unified relationship

23 that accounts for cars and buses because the classical MFD is not sufficient to capture

24 the traffic flow interactions of a multimodal traffic. The concept of passenger 25 macroscopic fundamental diagram ( $p$-MFD) is introduced. With this new relationship, 26 the efficiency of the global transport system, i.e. behaviors of cars and buses, can be 27 assessed. Intuitively, the p-MFD shape strongly depends on the mode ratio. Thus, user 28 equilibrium and system optimum are studied and compared. Finally, this relationship is 29 used to design bus system characteristics and to identify the optimal domains of 30 applications for different transit strategies. 


\section{INTRODUCTION}

34 Cities and transit agencies worldwide have to face an accelerating demand for mobility 35 as people continue to flock to urban areas seeking access to greater economic, educational, and social opportunities. This poses a challenge to optimally distribute city space to multiple transportation modes. To this end, management strategies have to be dynamic, multiscale, and simultaneously applied to individual cars and other transportation modes (such as public transport).

The core element of such management strategies is a global evaluation function of the transportation network. This function must quantify the performance of the whole system that can combine different transportation modes (individual cars, buses, trams, trucks, etc.). This is thus a challenge to capture the traffic dynamics of a complex network mixing these modes. It turns out that cities are complex and intricate systems. Therefore, they are impossible to model in perfect detail. The approach taken in this paper is to look at the transportation network at a macroscopic level. It is important to notice that the approach of the paper is very idealized. Indeed, the challenge here is to propose a modeling framework as general as possible. Then, it could be applied to a relatively wide range of situations and refined based on the characteristics of these situations.

To this end, we resort to an aggregated and parsimonious model to evaluate the transportation network performance. Such a model provides a better understanding and valuable insights on arterial traffic dynamics. The macroscopic fundamental diagram (MFD) can play this role. Indeed, on their seminal works (Godfrey, 1969; Mahmassani et al., 1984; Daganzo, 2007; Geroliminis and Daganzo, 2008), the authors pointed out a major insight: the MFD is an intrinsic property of the network itself and remains invariant when demand changes. The MFD is thus a reliable tool for traffic agencies to manage and evaluate solutions to improve mobility. (Haddad and Geroliminis, 2012; Keyvan-Ekbatani et al., 2012; Aboudoulas and Geroliminis, 2013; De Jong et al., 2013; Chiabaut, 2014; Haddad and Shraiber, 2014; Ramezani et al., 2015) furnished a very good example of how MFDs can be used to model and quantify ex ante effects of control.

64 Moreover, recent works (Boyaci and Geroliminis, 2011; Geroliminis and Boyaci, 2012; 
Leclercq and Geroliminis, 2013; Xie et al., 2013) propose an accurate method to analytically estimate the MFD for an arterial based on its characteristics (number of lanes, traffic signal parameters, etc.) and the characteristics of the public transport system (Chiabaut et al., 2014).

However, one of the remaining lacks of the MFD is that it only expresses the performance of the system as far as vehicles are concerned. Consequently, the average number of passengers present in each transport mode is not taken into account. Eichler and Daganzo (2006) presented the first instance trying to overcome this drawback. They seek to calculate average the pace for each mode. However, the number of passengers is roughly accounted for and the analysis stays very qualitative according to the authors themselves. Thus we propose in the paper to extend the concept of MFD in order to take into account the number of passengers using the transportation network and not only the number of vehicles. This new relationship is called the passenger Macroscopic Fundamental Diagram (p-MFD). Zheng and Geroliminis (2013), and Chiabaut et al. (2014) have simultaneously introduced the first principles of this relationship.

The mode choice of travelers should be considered as well. It is intuitive that the effect of the ratio of people using public transport rather than individual cars will impact the performance of the transportation network. The p-MFD makes it possible to address this issue and to understand how traffic conditions are modified by the mode choice of passengers. Different equilibriums can be investigated, notably user and system optimums. The ultimate goal of research towards this direction is to develop a strategy that makes people switch from a mode to the other.

The paper is organized as follow: Section 2 introduces the notion of passenger macroscopic fundamental diagram (p-MFD). Section 3 deeply investigates the impacts of modal choice and makes it possible to analytically compare user and system optimums. Section 4 focuses on the application of the p-MFD to network transportation services optimization. Finally, Section 5 proposes a discussion. 
In this first section, we extend the definition of MFD to propose a unified relationship that combines all the modes of an urban transportation network: cars, buses, metro, etc. The idea is to relate the number of passengers within the network to space-mean speed of these passengers according to a specific mode choice model. For the sake of simplicity, we first focus on two modes only: individual cars and buses. It is intuitive that, even in this simplest case, the classical MFD is not sufficient to evaluate the performance of the whole network because a bus counts for a unique vehicle. Notice that the methodology presented hereafter is general and can be extended to multiple modes.

\subsection{Case study}

As mentioned earlier, recent works suggest that there is a consistent relationship between the average network vehicle density and average network flow. Such a relationship is called a MFD. Consequently, we consider in the remainder of the paper an idealized city. Roads shape a very meshed urban network with signalized intersections

114 (see Figure 1a). We also assume that the flows are uniformly distributed among origins and destinations (Leclercq et al., 2014). Under this assumption, car traffic dynamics is well reproduced by a MFD $q(k)$ giving the space-mean flow of cars $q_{c}=q(k)[\mathrm{veh} / \mathrm{h}]$ on each link as a function of the space-mean density of the links within the city $k[\mathrm{veh} / \mathrm{km}]$

118 (see Figure 1b). Notice that the MFD can now be easily estimated accounting for the 119 effect of buses and control strategies (Geroliminis and Boyaci, 2012; Leclercq and 120 Geroliminis, 2013; Chiabaut et al., 2014). It is also worth noticing that the lower case 121 letters refer to variables expressed in terms of vehicles whereas the upper case letters 122 correspond to variables expressed in terms of passengers. We also assume that the maximal occupancy of a car is $\rho_{c}$ [pax/veh]. Notice that, for a realistic purpose, we consider that the maximal occupancy is equal to the observed average occupancy $(1.2$ pax/veh) rather than the effective maximal occupancy (5 pax/veh). We first assume a

126 trapezoidal car MFD for the sake of simplicity. This form is convenient to coarsely mimic 127 the influence of traffic signals. The parameters are the free-flow speed $u[\mathrm{~km} / \mathrm{h}]$, the critical speed $u_{c}$, the maximal flow capacity $q_{x}$ and the jam density $k_{x}$. The congested 
wave speed is denoted $w=u \cdot q_{x} /\left(u \cdot k_{x}-q_{x}\right)$. We assume that all the links are composed of $n$ 130 lanes $(n=2)$.

(a)

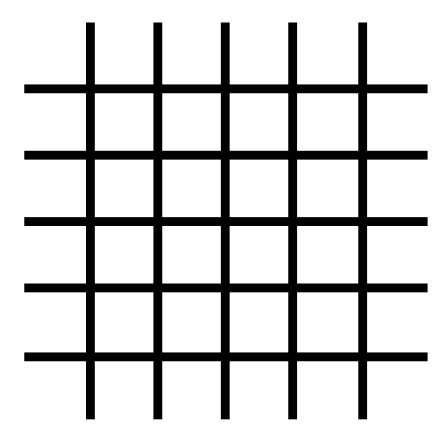

132

133

134

135

136

137

138

139

140

141

142

143

144

145

146

147

148

149

Figure 1: (a) a meshed urban network and (b) its associated car-MFD

(b)

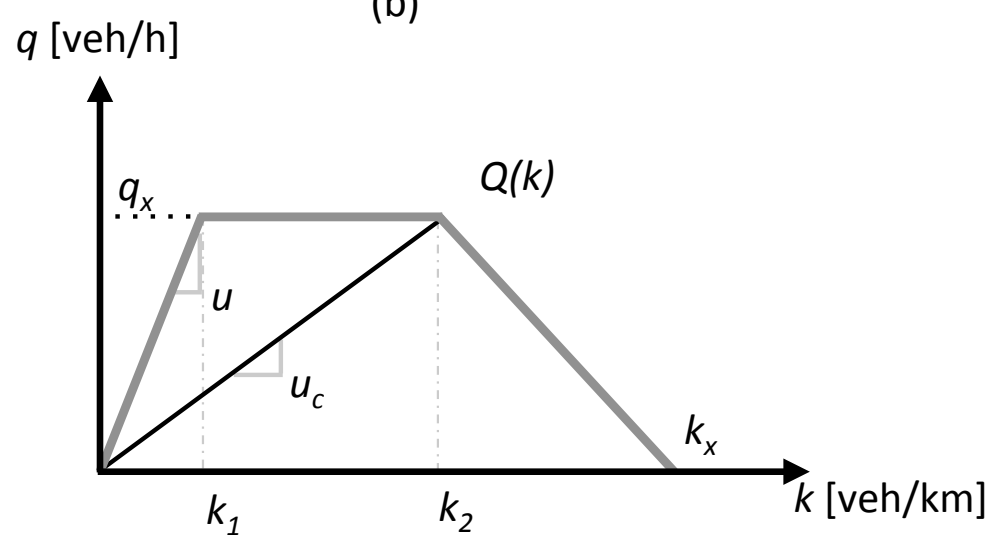

We first consider that the public transport system is only composed of buses that share the same roads as the car traffic. We also assume that trips of users can always be realized either by individual car or public transport system. The transit system is characterized by the bus time-headway $h[\mathrm{~h}]$ and the maximal speed of the buses $u_{t}$. We also assume that the maximal occupancy of a bus is $\rho_{t}$ [pax/bus] and that the buses are mixed with car traffic and no lanes are dedicated to them. Moreover, we consider that the average occupancies of both modes and the number of buses in operation do not depend on the traffic conditions and the mode choice. It turns out that, for a given timeheadway $h$ the number of buses $n_{\text {bus }}$ in operation is $n_{\text {bus }}=L /\left(h . v_{t}\right)$ (Hans et al., 2014) where $L[\mathrm{~km}]$ is the length of the bus lines of the transportation network and $v_{t}$ the average speed of the transit system. Physically, it corresponds to static timetables. Finally, Table 1 provides a nomenclature for the different variables and main parameters utilized in the paper. It also provides the values that have been used to draw illustrations.

\begin{tabular}{lll}
\hline Variable & Description & Value (if constant) \\
\hline$q$ & Car MFD [veh/h] & - \\
$q_{c}$ & Flow of cars $[\mathrm{veh} / \mathrm{h}]$ & - \\
$k_{c}$ & Density of cars $[\mathrm{veh} / \mathrm{km}]$ & - \\
$\rho_{c}$ & Average occupancy of cars [pax/veh] & 1.2 \\
$u$ & Free-flow speed of cars $[\mathrm{km} / \mathrm{h}]$ & 50 \\
\hline
\end{tabular}




\begin{tabular}{|c|c|c|}
\hline$u_{c}$ & Critical speed of cars $[\mathrm{km} / \mathrm{h}]$ & 7.7 \\
\hline$v_{c}$ & Average speed of cars $[\mathrm{km} / \mathrm{h}]$ & - \\
\hline$q_{x}$ & Maximal capacity of cars [veh/h] & 4250 \\
\hline$k_{x}$ & Jam density of cars [veh/km] & 450 \\
\hline$k_{1}, k_{2}, k_{l}$ & Specific densities of cars [veh/km] & $79,214,100$ \\
\hline$w$ & Congested wave speed $[\mathrm{km} / \mathrm{h}]$ & 18 \\
\hline$n$ & Number of lanes per link [lanes] & 3 \\
\hline$h$ & Bus time-headway [h/bus] & - \\
\hline$\rho_{t}$ & Maximal occupancies of bus [pax/bus] & 40 \\
\hline$n_{\text {bus }}$ & Number of bus in operation [bus] & - \\
\hline$L$ & Length of the transit system $[\mathrm{km}]$ & 10 \\
\hline$v_{t}$ & Average speed of the transit system $[\mathrm{km} / \mathrm{h}]$ & - \\
\hline$u_{t}$ & Free-flow speed of the transit system $[\mathrm{km} / \mathrm{h}]$ & 36 \\
\hline$P$ & Total flow of the system [pax/h] & - \\
\hline$F_{c}$ & Flow of passengers using cars [pax/h] & - \\
\hline$F_{t}$ & Flow of passengers using transit [pax/h] & - \\
\hline$K$ & Total density of the system $[\mathrm{pax} / \mathrm{km}]$ & - \\
\hline$K_{c}$ & Density of passengers using cars [pax $/ \mathrm{km}]$ & - \\
\hline$K_{t}$ & Density of passengers using transit [pax $/ \mathrm{km}]$ & - \\
\hline$\tau$ & Mode choice ratio [\%] & - \\
\hline$\alpha$ & Ratio of the network dedicated to transit [\%] & - \\
\hline
\end{tabular}

154 To introduce the p-MFD, the flow is now expressed in terms of passengers per time 155 [pax/h]. Let $P$ denote this flow. $P$ is equal to the sum of passengers using cars $F_{c}$ and

Table 1: List of main variables and parameters

\subsection{The p-MFD} passengers using transit system $F_{t}$. It is worth noticing that $F_{c}$ directly derives from the car MFD $q(k)$ while $F_{t}$ must be obtained from the characteristics of the transit system. Moreover, as in the classical definition of the MFD, it is thus really appealing to link the flow to the average density of passengers in the city $K$. However, this is not trivial because $K$ has to be expressed in terms of passengers per space [pax/km]. Moreover, it turns out that the mode choice of passengers between cars and buses has an impact on the flow and density. This ratio is denoted $\tau$ and is equal to $K_{c} / K$ where $K_{c}$ is the density of passengers using cars. 
165 In this section, $\tau$ is considered as static, i.e. constant in time and independent of traffic conditions. Consequently, $\tau$ is exogenously given. Physically, it corresponds to an equilibrium situation where day after day the same demand and traffic conditions occur.

168 Notice that these assumptions are relaxed and studied in the forthcoming section.

169 Consequently, the total average flow and the total average density are:

$$
\begin{gathered}
P(K)=F_{c}\left(K_{c}\right)+F_{t}\left(K_{t}\right) \\
K=K_{c}+K_{t}
\end{gathered}
$$

171 Where $K_{c}$ and $K_{t}$ are respectively the average density in passengers of cars and buses.

172 It is thus appealing to link $P$ with $K$ and to correctly determine the function $P(K)$ and 173 especially its shape. Consider a given density of passengers $K$. According to the

174 definition of the mode ratio $\tau$, the density of passenger in cars is $K_{c}=\tau . K$ [pax $\left./ \mathrm{km}\right]$. It is 175 worth noticing that this density is also equal to the density of cars weighted by the 176 average occupancy, i.e. $k_{c}=K_{c} / \rho_{c}$ where $k_{c}$ is the density expressed in [veh $\left./ \mathrm{km}\right]$. It makes 177 it possible to calculate the average flow in terms of passengers: $F_{c}=\rho_{c} q\left(k_{c}\right)=\rho_{c} q\left(K_{c} / \rho_{c}\right)$ 178 where $q$ is the car MFD. It comes that the associated average speed of passengers using 179 cars is $v_{c}=q_{c} / k_{c}$.

181 Concerning the transit system, the density of bus passengers is given by $K_{t}=(1-\tau) \cdot K$ and 182 cannot exceed $\rho_{t} . n_{\text {bus }} / L$ the maximal density of the transit system. Consequently, the 183 associated flow is thus equal to $F_{t}=\min \left((1-\tau) K, \rho_{t} n_{b u s} / L\right) / h$. Because we assume 184 that the bus fleet size $n_{b u s}$ is constant, i.e. it does depend on traffic conditions, $h$ directly 185 depends on the average speed of the transit system: $h=L /\left(n_{\text {bus. }} v_{t}\right)$. Consequently, when 186 the car traffic does not constrain the buses, bus speeds are always equal to the free flow 187 speed $u_{t}$ and the headway $h$ is not degraded. However, in the case of mixed traffic, 188 congestion may impact the bus system when the speed of the car $v_{c}$ is lower than the 189 maximal speed of the bus $u_{t}$. It comes that $h=L / n_{b u s} . \min \left(u_{t}, v_{c}\right)$ where $v_{c}$ is the speed 190 of the cars: $v_{c}=q_{c} / k_{c}$.

192 Finally, equation (1) can be expressed as:

$$
P(K)=\rho_{c} q\left(\frac{\tau K}{\rho_{c}}\right)+\frac{1}{L} \cdot \min \left((1-\tau) K \cdot L, \rho_{t} n_{b u s}\right) \cdot \min \left(u_{t}, v_{c}\right)
$$


195 Figure 2 presents the results. The thick gray line corresponds to the car MFD whereas the thin black lines are the different p-MFDs. It is thus appealing to observe the impacts of the bus systems characteristics on the p-MFD shape. To this end, Figure 2a pinpoints the sensitivity of the p-MFD to the bus time-headways $h$. For this application, all the variables are assumed constant except $K$ (and $P$ ) and $h$ that ranges between $3 \mathrm{~min}$ and 12 min. It turns out that a maximal capacity can be reached for a specific value. Notice that the effect of the bus system on the car traffic dynamics is not accounted here, i.e. the formulation of the car MFD does not depend on the time-headway $h$. For a given car MFD, it is thus possible to determine the optimal $h$ to maximize the transportation system performance. In the same vein, Figure $2 \mathrm{~b}$ highlights the impacts of $\tau$ on the pMFD shape. Consequently, the only variables that change are $K$ (and $P$ ) and $\tau$ that ranges between 0.1 and 1 . It is not surprising that the performance can also be optimized for a specific value of $\tau$. It is thus appealing to study in details the impacts of $\tau$ and to focus on dynamic $\tau$. This will be investigated in the next section.

(a)

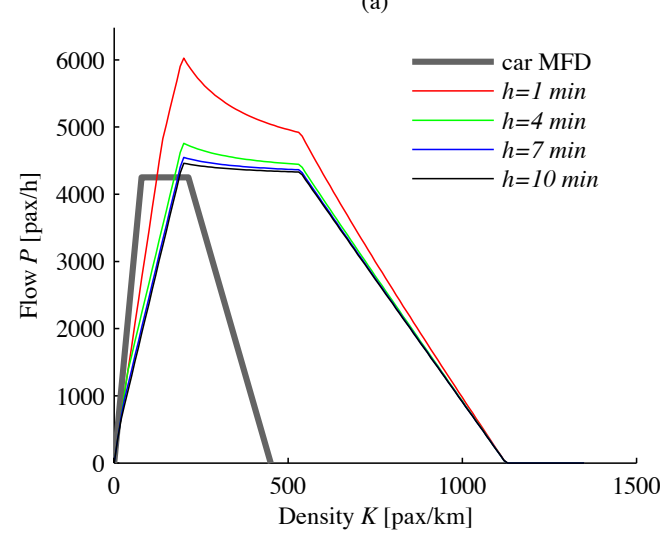

(b)

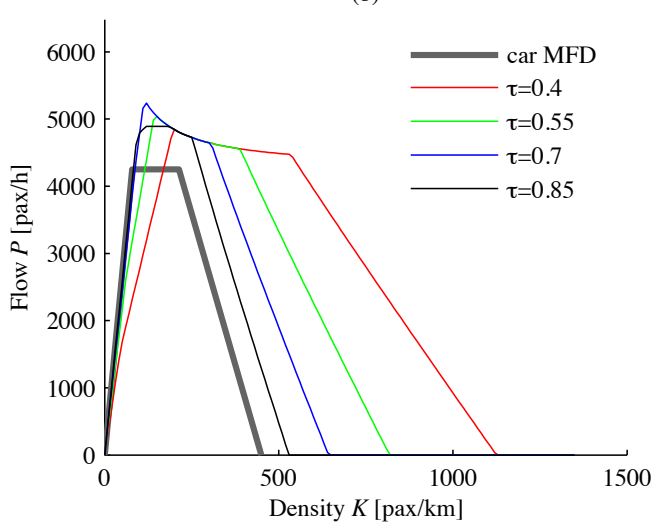
and (b) sensitivity to mode ratio $\tau$

216 Now that we have introduced the concept of p-MFD, this section focuses on the effects of 217 the mode choice and studies the associated equilibrium of the transportation network. 218 Notably, system and user optimums are investigated. To this end, we now consider that the mode choice is based on the utility of the mode. The utility is expressed as the travel cost of using that mode at the beginning of the trip. The travel cost of the whole route 
221 consists of travel time for cars and buses. This very naïve assumption can be refined 222 without changing the general methodology presented hereafter.

\subsection{System optimum}

We first focus on the system optimum. It corresponds to the second principle of 227 Wardrop. In such an equilibrium situation, the average journey time is minimum, i.e. the average speed of passengers is maximal. It is worth noticing that the speed is equal to the ratio of the demand with the density. Consequently, for a given density $K$, the associated flow $P$ must satisfy the following equation to maximize the average speed:

$$
P(K)=\max _{\tau}\left[\rho_{c} q\left(\frac{\tau K}{\rho_{c}}\right)+\frac{1}{L} \cdot \min \left((1-\tau) K \cdot L, \rho_{t} n_{b u s}\right) \cdot \min \left(u_{t}, v_{c}\right)\right]
$$

232 Based on this equation, we are now able to determine the function $P$ for all the possible traffic conditions, i.e. all the possible values of $K$.

The free-flow conditions correspond to the situations where the total passenger demand is satisfied by the system. The p-MFD is directly obtained by solving equation (3). Figure 3a presents the resulting p-MFD in case of a trapezoidal car MFD. It turns out that the passenger mode allocation $\tau$ is not constant. This is confirmed by Figure $3 \mathrm{~b}$ that shows the evolution of $\tau$ with respect to the passenger density level. Car is the unique mode until the density reaches the critical density $k_{1}$ (see Figure $1 \mathrm{~b}$ ), i.e. the demand will exceed the maximal car capacity $\rho_{c} q_{x}$. Then passengers have to switch from cars to the

244 transit system. Note that this corresponds to the optimal situation where passengers are ready to change mode rather than to degrade the traffic conditions. We do not focus in the paper on the possible policies to make users change mode. However, incentive or congestion pricing, traffic information, prescriptive management could be efficient and innovative solutions. 

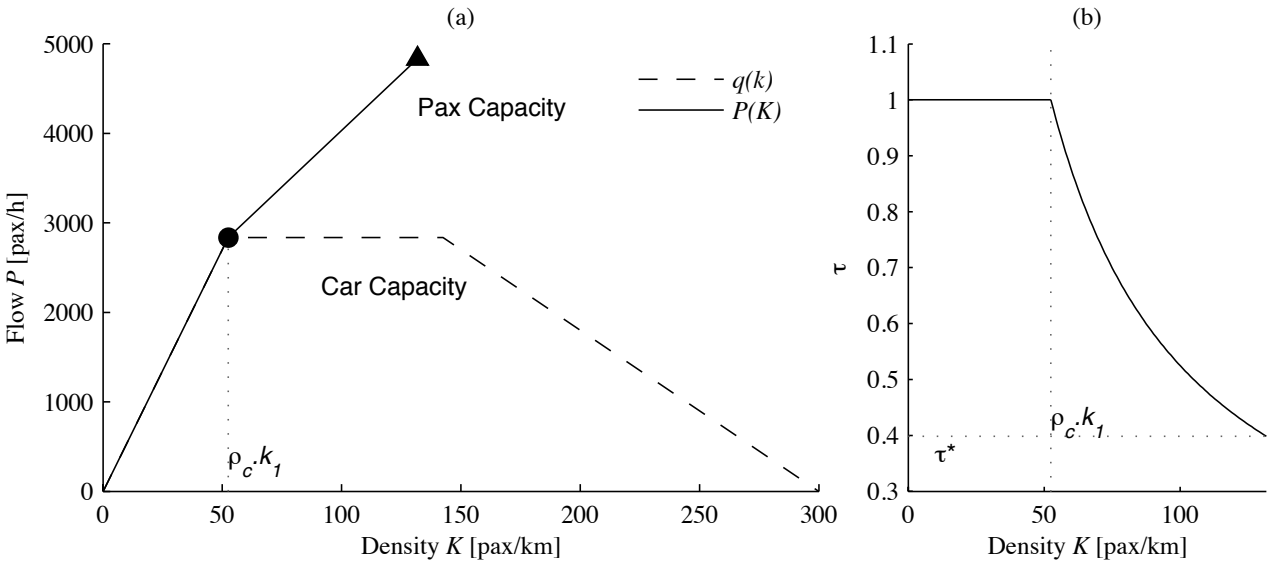

(c)
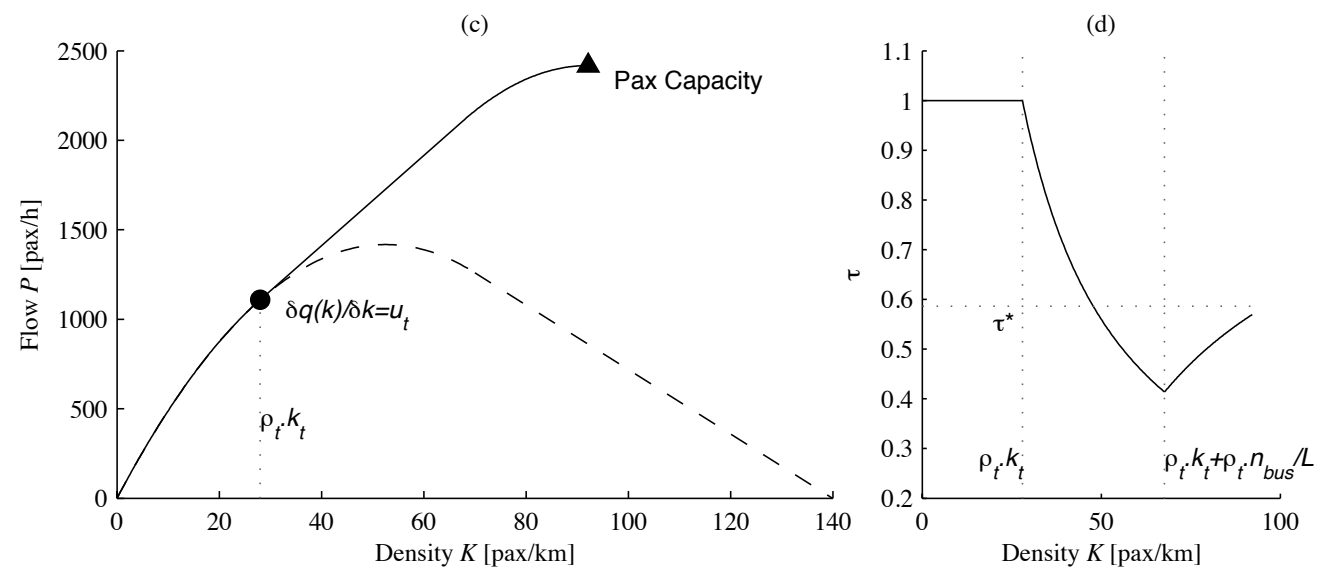

Figure 3: (a) free-flow part of the p-MFD for a trapezoidal MFD and (b) evolution of $\tau$ with $K$

(c) free-flow part of the p-MFD for a curved MFD and (d) evolution of $\tau$ with $K$

255 This method can be applied for any shape of MFD. However, calculations are more complicated. We now assume that the car MFD can take any concave shape. We consider here, for the example, a function composed of a parabolic and linear part (see Figure 3c):

$$
\begin{aligned}
& Q(k)=a k^{2}+b k \text { for } k<k_{l} \\
& Q(k)=w\left(k_{x}-k_{l}\right) \text { otherwise. }
\end{aligned}
$$

260 Where, $a=-u^{2} /\left(2 . q_{x}\right), b=u$ and $k_{l}=-(w+b) /(2 a)$. Such a formulation ensures to maintain the 261 same free-flow speed than the triangular car MFD.

262 To determine the associated p-MFD in case of SO, equation (3) has to be re-written as:

$$
K(P)=\min _{\tau}\left(K_{c}+K_{t}\right)
$$

264 It is also worth noticing that $K_{c}$ and $K_{t}$ (respectively) can be expressed as a function of $F_{c}$ 265 and $F_{t}$ (respectively). Let us consider $F_{c}^{*}$ and $F_{t}^{*}$ to be the optimal solution of (5) for a 266 given total density $K^{*}$. A small increment $\Delta F_{c}$ and $\Delta F_{t}$ of the flows $F_{c}$ and $F t$ will change 267 the density value. This change can be approximated by a first order Taylor expansion: 


$$
K\left(F_{c}^{*}+\Delta F_{c}\right)=K_{c}\left(F_{c}^{*}\right)+K_{c}^{\prime}\left(F_{c}^{*}\right) \cdot \Delta F_{c}
$$

$$
K_{t}\left(F_{t}^{*}+\Delta F_{t}\right)=K_{t}\left(F_{t}^{*}\right)+K_{t}^{\prime}\left(F_{t}^{*}\right) \cdot \Delta F_{t}
$$

Thus, the total variation of density is:

$$
\Delta K=\Delta P\left(K_{c}^{\prime}\left(F_{c}^{*}\right) \cdot \frac{\Delta F_{c}}{\Delta P}+K_{t}^{\prime}\left(F_{t}^{*}\right) \cdot \frac{\Delta F_{t}}{\Delta P}\right)
$$

272 This equation can be simplified because if we consider that buses are not affected by 273 traffic congestion, thus: $K_{t}^{\prime}\left(F_{t}^{*}\right)=1 / u_{t}$ because $K_{t}=n_{b u s} \cdot \rho_{t} / L, F_{t}=\rho_{t} / h$ and $n_{\text {bus }}=$ $274 L / h . u_{t}$. This is true because we only consider free-flow situations in this section of the 275 paper. It comes:

$$
\Delta K=\Delta P\left(\frac{\delta K_{c}}{\delta F_{c}}\left(F_{c}^{*}\right) \cdot \frac{\Delta F_{c}}{\Delta P}+\frac{1}{u_{t}} \cdot \frac{\Delta F_{t}}{\Delta P}\right)
$$

277

$\Delta K$ is given by the combination of $\frac{\Delta F_{c}}{\Delta P}$ and $\frac{\Delta F_{t}}{\Delta P}$ that minimizes the RHS of equation (8). This quantity admits a lower bound that is equal to $\frac{\delta K_{c}}{\delta F_{c}}\left(F_{c}^{*}\right)$ when $\frac{\delta K_{c}}{\delta F_{c}}\left(F_{c}^{*}\right)<\frac{1}{u_{t}}$ or to $\frac{1}{u_{t}}$ otherwise. Thus, it appears that when the total flow varies from $\Delta P$ the optimal solution of (8) is only modified for the car flow if $\frac{\delta K_{c}}{\delta F_{c}}\left(F_{c}^{*}\right)<\frac{1}{u_{t}}$ and for the transit flow otherwise. Consequently, passengers have to shift of mode when $\frac{\delta K_{c}}{\delta F_{c}}\left(F_{c}^{*}\right)=\frac{1}{u_{t}}$ to reach SO.

Based on these results, the p-MFDs for SO in free-flow conditions are highlighted in Figure 3c. The evolution of $\tau$ with respect to the demand level is slightly different from the trapezoidal MFD case, see Figure 3d. To obtain the SO solution, car is the unique mode until a certain car density value that corresponds to $k_{t}$ such as $\partial q\left(k_{t}\right) / \partial k=u_{t}$; then passengers switch to the transit system until all the buses are full; finally, the remaining car capacity is used until the system's capacity is reached.

\subsubsection{Congested conditions}

We now aim to determine the p-MFD when traffic is congested. For cars, the MFD directly accounts for this capacity reduction. For buses, they are not impacted for small congestion, i.e. when $F_{c}\left(K_{c}\right) / K_{c}>u_{t}$. However, when $F_{c}\left(K_{c}\right) / K_{c}<u_{t}$, the buses are slowed down by the queues. Characteristics of the transit system have to be dynamically modified according to $h=L / n_{\text {bus }} . \min \left(u_{t}, v_{c}\right)$. It makes it possible to account for congestion in the expression of $F_{t}$. Because car-MFD expressed in terms of passengers, 
i.e. $F_{c}$, also reproduces congested states, the congested part of the p-MFD can be determined according to equation (3). It turns out that equation (3) is only a maximization process that depends on the formulation of $F_{c}$ and $F_{t}$.

(a)

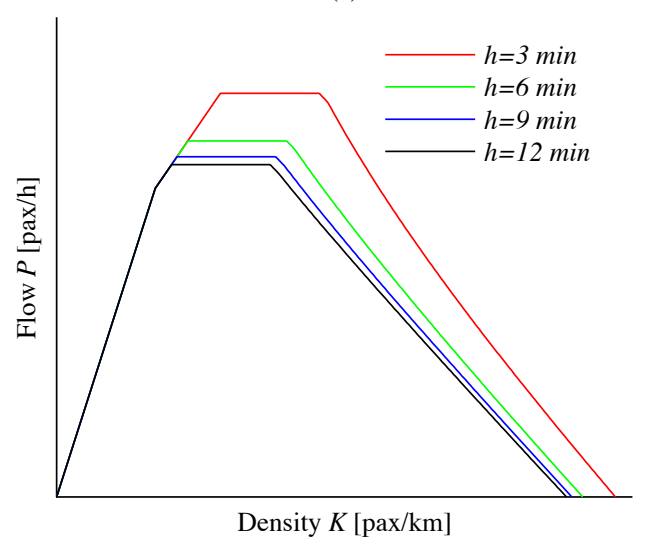

(b)

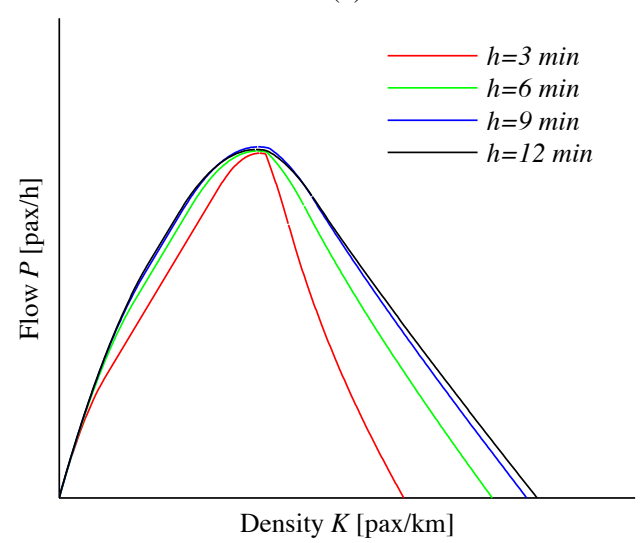

302

303

304

305

306 3.2.1. Wardrop's first principle situation. It will be studied in Section 4.

Figure 4: Complete p-MFD in mixed traffic for (a) a car trapezoidal MFD and (b) a car parabolic-linear MFD

Figure 4 presents the associated p-MFD. Figure 4a highlights the influence of the bus time-headways $h$ for a car trapezoidal MFD, i.e. all variables are constant except $h$ that ranges between $3 \mathrm{~min}$ and $12 \mathrm{~min}$. It is worth noticing that the transformation is always homothetic because of the linearity of the car MFD shapes. Indeed, for other concave shapes, Figure $4 \mathrm{~b}$ shows very different results. It is interesting to notice that a specific combination of bus time-headways and car MFDs leads to optimal situations. It is thus appealing to use the p-MFD to determine the optimal bus time-headway for a given 
324 The Wardrop's first principle (Wardrop, 1952) can be adapted to our multimodal case.

325 Each user non-cooperatively seeks to minimize his cost of transportation. Consequently,

326 the speeds in all modes actually used equal between them and are higher than those that

327 would be experienced by a single traveller on any unused mode. This principle is 328 referred to as user equilibrium (UE) in the remainder of the paper. Consequently, when 329 the transportation network satisfies the UE, passengers either use only the car or they use both modes.

Figure 5a shows the associated p-MFD in the case of car trapezoidal MFD. It turns out that is still an all-or-nothing situation. Car is the preferred mode until the arterial becomes congested and the speed of the cars becomes the same as the speed of the buses. Then, the passenger demand is split in both modes according to a ratio $\tau$. It is also worth noticing that the difference in terms of flow between the UE and SO is very high (see SO p-MFD in blue). Moreover, these differences occur in free-flow and lead to a capacity reduction in case of UE. It means that the performance of the transportation network can be deeply optimized by managing the demand rather the self-organization situation. Traffic management strategies must focus on free-flow situations that are really close to the system capacity.

343 Figure 5b highlights the p-MFD for a parabolic-linear car MFD. For this specific shape of 344 car MFD, differences between black and blue lines are smaller but the network performance can still be increased by changing the equilibrium from user to system 346 optimum.

(a)

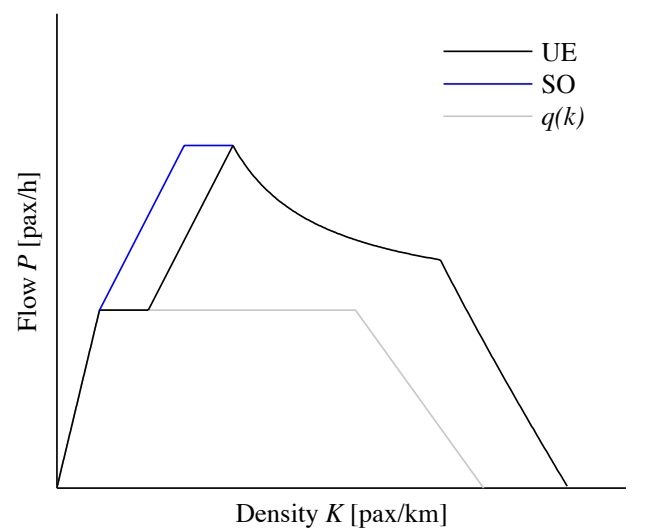

(b)

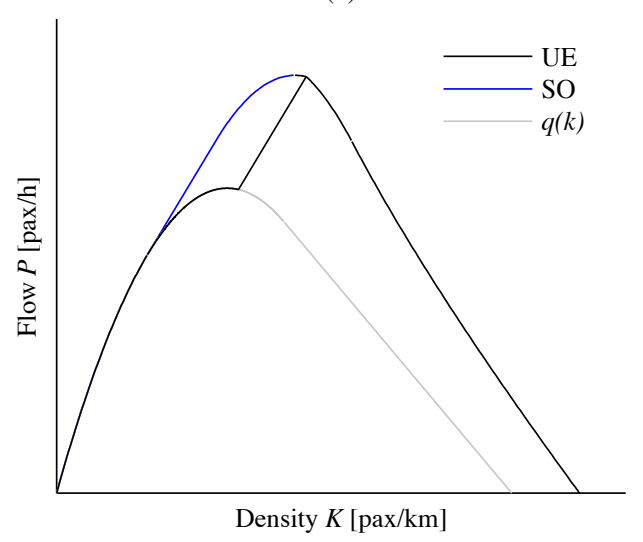


353 In the vein of Leclercq and Geroliminis (2013), the Logit model can also be easily 354 adapted to a multimodal traffic. The associated situation is referred to as stochastic user equilibrium (SUE) in the remainder of the paper. We now assume that the ratio of flows between cars and buses only depends on the difference in travel times, i.e. speed, between both modes:

$$
\frac{F_{t}}{F_{c}}=e^{-\theta\left(\frac{L}{v_{t}}-\frac{L}{v_{c}}\right)}
$$

Where $\theta$ is the parameter of the Logit model and $L$ the average trip length.

We have now all the equations to formulate a parametric expression of the flow $P$ for the arterial with respect to $v_{c}$. This defines a simple method to calculate the p-MFD for the Logit model. Figure 6 presents the resulting p-MFD. Notice that all the variables are constant except $\theta$. Therefore, we have tested several $\theta$ values (from 0.05 to 0.9 with an increment of 0.05). It turns out that p-MFDs fall between UE and SO. Moreover, UE, SUE and SO only differ at the network level in free-flow situations when average speed is comprised between $u$ and $u_{t}$ for trapezoidal MFD and $u_{s}$ and $u_{t}$ for curved MFD $\left(u_{s}\right.$ such as $\frac{\delta F_{c}}{\delta K_{c}}=u_{t}$ ). This is not surprising because in congestion both modes have the same speed. The mode ratio is then constant.

(a)

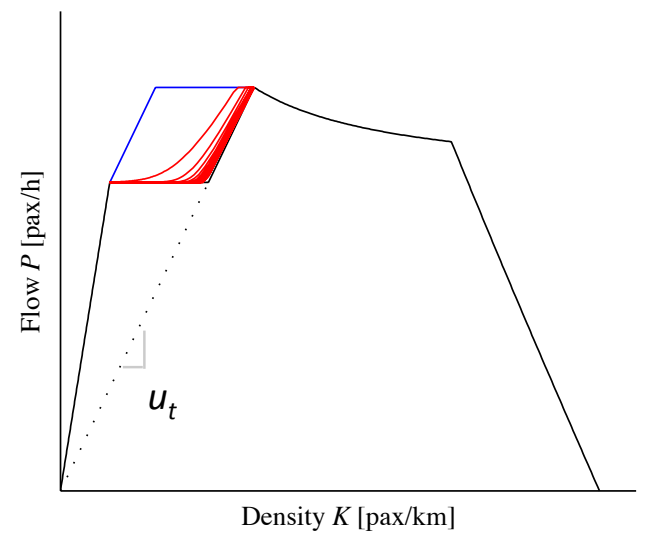

(b)

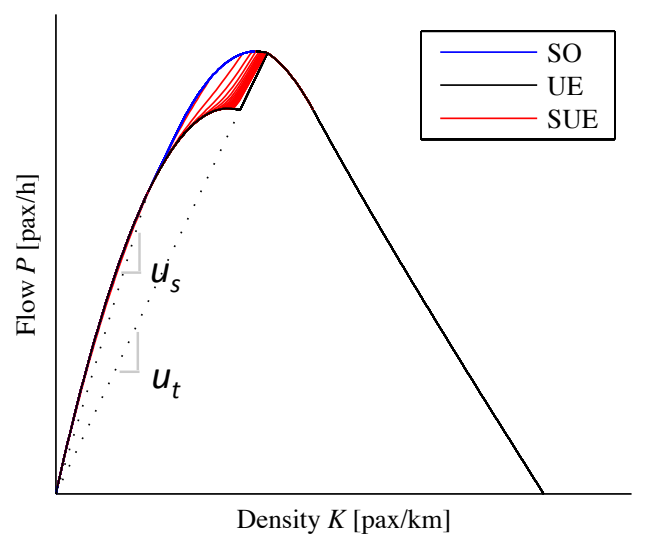

Figure 6: Comparison of SUE, UE and SO in case of (a) a car trapezoidal MFD 
378 Even if rough comparisons between UE and SO are proposed by Figure 5a, it is still appealing to study these difference in more detail. To this end, we now focus on the marginal costs of UE and SO. This will give useful insights on the pricing policy that can be implemented to adapt the costs of each mode and switch the transportation network from UE to SO.

The marginal cost of a mode defines the increase of the cost of this mode if one more passenger chooses to use this mode. The marginal cost of mode $i$ is obtained by differentiating the cost function $c_{i}\left(K_{i}\right)$ :

$$
M C_{i}\left(K_{i}\right)=c_{i}\left(K_{i}\right)+K_{i} c_{i}^{\prime}\left(K_{i}\right)
$$

388 Here the cost of a mode is characterized by the average speed, i.e. $c_{i}=F_{i}\left(K_{i}\right) / K_{i}$. Thus, the derivative $c_{i}^{\prime}$ can be formulated as:

$$
c_{i}^{\prime}\left(K_{i}\right)=\frac{K_{i} F^{\prime}\left(K_{i}\right)-F\left(K_{i}\right)}{K_{i}^{2}}
$$

Finally, the marginal costs of each mode are equal to:

$$
\begin{aligned}
& M C_{c}\left(K_{c}\right)=F_{c}^{\prime}\left(K_{c}\right)=\frac{\delta F_{c}\left(K_{c}\right)}{\delta K_{c}} \\
& M C_{t}\left(K_{t}\right)=F_{t}^{\prime}\left(K_{t}\right)=\frac{\delta F_{t}\left(K_{t}\right)}{\delta K_{t}}
\end{aligned}
$$

393

With this pricing, SO is reached by letting the system self-organized, i.e. Wardrop's user equilibrium. The conditions of SO highlighted in the previous section can be identified. It turns out that the demand is entirely assigned to the individual vehicle mode until $\frac{\delta F_{c}\left(K_{c}\right)}{\delta K_{c}}=\frac{\delta F_{t}\left(K_{t}\right)}{\delta K_{t}}$. It is also worth noticing that dynamic pricing is required to reach system optimum because $\frac{\delta F_{c}\left(K_{c}\right)}{\delta K_{c}}$ changes with $K_{c}$. Especially, the pricing is lower when traffic becomes saturated due to the concave shape of $F_{c}\left(K_{c}\right)$.

\section{APPLICATION TO TRANSPORTATION NETWORK SERVICES OPTIMIZATION}

The section aims to use the p-MFD as a powerful tool to compare and design optimal control strategies. Firstly, we assume that $\tau$ can depend on the passenger demand, i.e. $\tau$ is dynamic. According to this assumption, it makes it possible to compare the upper envelope of the associated p-MFD. Secondly, p-MFDs are used to investigate how DBLs 
impact the transportation system performance and may be a powerful tool to increase capacity even in UE case.

When buses and cars share the same network, buses tend to constrain traffic flow because of their lower speed. They may act as moving bottleneck that create local capacity reductions. Intuitively, the occurrence of these reductions increases with the frequency of the bus system (Xie et al., 2013). Consequently, an acceptable trade-off between the bus frequency and the impact on the capacity of the car-MFD must be determined to ensure an efficient performance of the transportation network.

To cope with this issue, the p-MFD is an adapted tool to calculate the optimal bus timeheadway to reach the system optimum. To this end, the car MFD now accounts for the effects of buses. $q(k)$ is parameterized by the bus system characteristics. Figure $7 \mathrm{a}$ highlights the influence of the bus time-headways on car MFD. As previously mentioned, an increase of $h_{\text {bus }}$ reduces the capacity for cars. To mimic this influence, the maximal capacity of cars $q_{x}$ now depends on the headway:

$$
q_{x}(h)=\left(n-e^{1-\frac{h}{h_{m}}}\right) \cdot q_{x} / n
$$

where $h_{m}$ is the minimal acceptable headway (here $h_{m}=1 \mathrm{~min}$ ). Notice that we can obtain more realistic MFD estimates by extending the work of Boyaci and Geroliminis (2011) and Xie et al. (2013) to the network level. However, this section only aims to introduce the general methodology to determine optimal bus time-headways.

430 Car MFDs are now directly related to the value of the bus time-headway $h$, see dotted lines in Figure 7a. These lines correspond to $h$ ranged between $1 \mathrm{~min}$ and $30 \mathrm{~min}$. Notice that the number of bus in operation $n_{\text {bus }}$ changes with $h$ but remains independent to the traffic conditions for a given $h$. Moreover, Figure 7a also shows the associated p-MFDs in

434 case of SO situation. This is clearly the most pertinent case to address for a city or transit manager. For a given passenger demand, managers seek to maximize the average speed on the transportation network, i.e. to minimize the average density. The upper envelope $U p$ of the calculated p-MFDs corresponds to the set of the optimal situations: 


$$
U_{p}(K)=\max _{K}[P(K)]
$$

440 It ensures that the average speed is always maximal. Figure 7a highlights in red this 441 upper envelope. Consequently, p-MFD provides the optimal bus time-headway with 442 regard to the passenger demand. Figure $7 \mathrm{~b}$ depicts the evolution of the optimal bus 443 time-headway with the passenger density. It turns out that very high bus frequencies 444 are required to reach high capacities. Unfortunately, such frequencies are very difficult 445 to maintain in practice. It is also worth noticing that the assumptions made to account 446 for the effects of bus in car MFD formulation strongly impacts the results. However, the 447 methodology proposed here can also be applied for a more realistic car MFD coming 448 from simulation as in (Chiabaut, 2014).

(a)

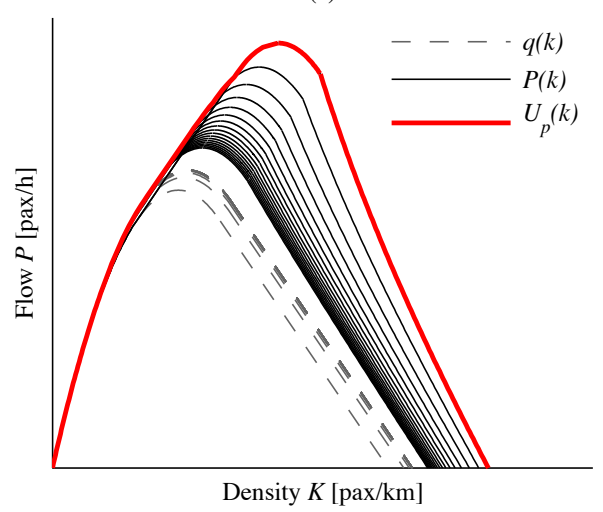

(b)

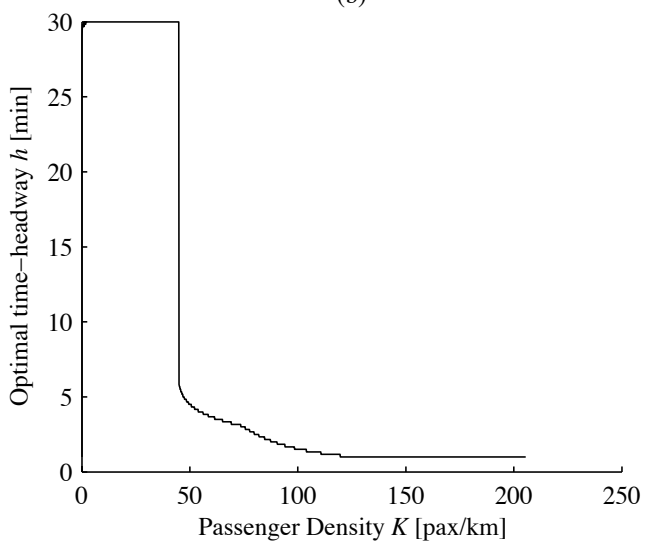

450

451

452

453

454

455

456

457

458

459

460

461

Figure 7: (a) Impact of the bus system on car MFD (b) upper bound of the transportation system

\subsection{Comparison of control strategies}

After considering only mixed traffic stream, we seek now to incorporate DBL in the formulation of the p-MFD. The final goal is to compare different traffic management strategies to improve the transportation network performance. Consequently, we only consider user equilibrium situations in this section of the paper.

The creation of DBL within the network engenders a capacity reduction for the cars. To make this phenomenon explicit, consider here that $\alpha$ is the ratio of lanes fully dedicated to a rapid public transport mode such as buses or trams. As Gonzales and Daganzo 
463 (2012), we assume that the car MFD is homogenously reduced of $\alpha$ times its original 464 formulation:

$$
q_{D B L}(k)=\alpha q(k)
$$

As previously mentioned, the works of (Xie et al., 2013) can be easily adapted to estimate a more accurate MFD. Because these considerations are out of the scope of the paper, the maximal capacity for cars is now equal to $\alpha . q_{x}$ and the critical speed $u_{c}$ remains constant for both cases of trapezoidal and curved car MFD.

We can theoretically segregate the public transport system into two parts: (i) a rapid transit system that can use the DBL network and (ii) the remainder of the fleet. Notice that the fleet size is equal for both studied cases. To mimic the effects of DBL on the transit system, we consider that the average speed of vehicles (buses or trams) using the DBL is increased. From a macroscopic lens, they have an average speed $u_{t}^{1}>u_{t}$. The fleet of the buses that cannot use the DBLs keeps an average speed equal to $u_{t}$.

We apply equation (3) to determine the associated p-MFD in the case of UE. Figure 8 presents the results. Notice that the fleet size is assumed constant and the only changing variables are $K$ (and $P$ ) and the $\alpha$ ratio that ranges between 0.5 and 1 . Figure 8 a 482 highlights the main difference between DBL and mixed cases ( $\alpha$ is equal to 0.8 ). It is not surprising to observe that rapid system is competitive before the remainder of the transit system because of their higher average speed. Thus individual car is the only used mode until the speed is reduced to the average speed of rapid system $u_{t}^{1}$. The remaining of the bus fleet becomes advantageous when the situation is enough congested, see the DBL optimal area in Figure 8a. Notice that the switching traffic conditions are directly given by the speed of the different modes. This process can be extended to any number of modes. It is also worth noticing that, even in a very congested situation, the flow of the p-MFD associated to the DBL case is never null. Indeed, we have assumed that the DBLs are never blocked by traffic congestion. 
(a)

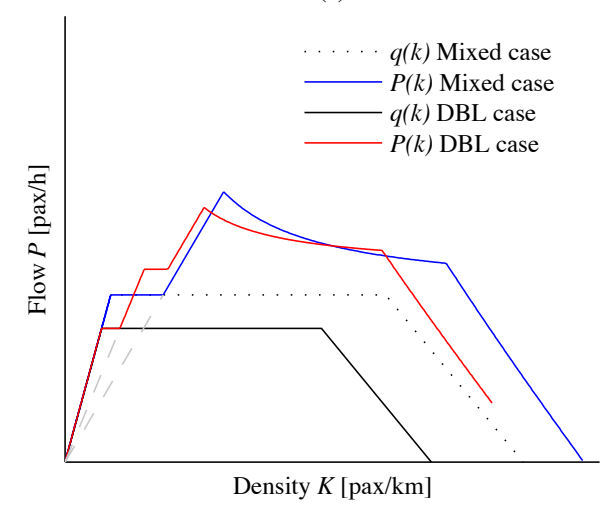

(b)

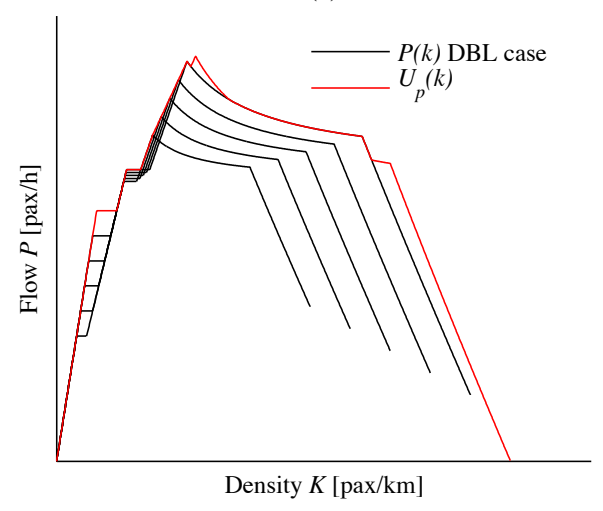

(c)

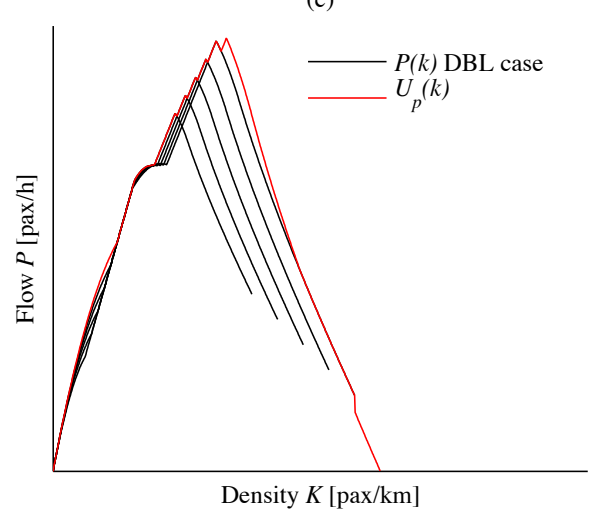

Figure 8: (a) Impact of DBL creation in case of UE and optimal domains of application for (b) a car trapezoidal MFD (c) a parabolic-linear MFD

Finally, we can identify the optimal domains of application in case of UE. As previously mentioned, optimal domains are determined by identifying solutions that maximize the flow for a given density, i.e. maximize the average speed. The red curves depict the upper bound of p-MFD calculated for $\alpha$-values comprised between 0.5 and 1 . In the simplest case of trapezoidal car-MFD, Figure $8 \mathrm{~b}$ highlights that the creation of DBLs can increase the capacity in free-flow conditions. It is also worth noticing that p-MFD for DBL case never reached a null-flow in congested situations. Indeed, DBL ensures that the bus system can still operate even in very congested states. Similar observations can be formulated in the case of curved-MFD, see Figure 8c. It turns out that DBL can be an optimal strategy in case of UE. This is very promising because global transportation network performance can be increased by promoting public transport even in the case of UE. 
513 This paper developed tools to analytically assess the performance of a multimodal

514 transportation network. To this end, the paper extends the MFD definition to account for

515 the average number of passengers in each mode. The objective is to obtain a unique 516 function to determine the domains of relevance of different transit strategies, where the 517 system cost is minimized.

519 First analytical considerations introduce the concept of p-MFD and study its sensitivity 520 to the bus system characteristics in case of a static mode choice. Then, the assumption is 521 relaxed to unveil the impacts of the mode choice on the transportation network 522 performance. Consequently, the user equilibrium case can be compared to the system 523 optimum situation.

525 This theoretical canvas can then be used to cross compare different transit strategies 526 and to design the optimal bus system characteristics. Especially, the paper focuses on 527 determining the more efficient bus time headway in case of mixed traffic. Then, the 528 study is devoted to the introduction of DBL. The p-MFD permits to determine the 529 optimal domains of application of DBL.

531 We acknowledge that the approach proposed in the paper is highly conceptual and 532 applied to a very idealized network. However, such an approach makes it possible to 533 provide a general modeling framework that can then be adapted to a large range of 534 situations. Nonetheless, this idealized analysis provides insights into how to assess the 535 global performance of a multimodal transportation network and how to compare 536 different traffic management strategies.

538 Finally, the results of this paper can be generalized for any design of the network. One of 539 the next extensions is to deal with a spatial distribution of traffic conditions on the 540 network. Indeed, the assumption of uniform distribution of flows can be relaxed 541 allowing for heterogeneous OD demands and mode choice ratio. Moreover, the work can 542 be extended to account for the feedback on the multimodal demand. Indeed, a fixed 543 demand has been considered in the paper but traffic conditions may induce less or more 
544 demand that have to be accounted for when calculating the p-MFD. More realistic car 545 MFD formulation can also be considered by resorting to simulation as in (Chiabaut et al., 546 2014) or more sophisticated estimation method (Hans et al., 2014a). Finally, a last step 547 will be to estimate the p-MFD from field data. This task clearly requires very detailed 548 data (passenger counts, vehicle occupancies, OD matrix, etc.). Urban mobility simulation 549 software may provide synthetic but insightful measurements to estimate more realistic 550 p-MFD. 
552

553

554

555

556

557

558

559

560

561

562

563

564

565

566

567

568

569

570

571

572

573

574

575

576

577

578

579

580

581

582

583

\section{REFERENCES}

Aboudolas, K., Geroliminis, N., 2013, Perimeter and boundary flow control in a multireservoir heterogeneous networks, Transportation Research Part B 55, 265-281.

Boyaci, B., Geroliminis, N., 2011, Estimation of the network capacity for multimodal urban systems, Procedia - Social and Behavioral Sciences 16, 806-813.

Chiabaut, N., 2014, Investigating Impacts of Pickup-Delivery Maneuvers on traffic flow dynamics, Procedia - Social and Behavioral Sciences, to be published.

Chiabaut, N., Xie, X., Leclercq, L., 2014, Performance analysis for different designs of a multimodal urban arterial, Transportmetrica B: Transport Dynamics, 2(3), 229-245.

Daganzo, C.F., 2007, Urban gridlock: macroscopic modeling and mitigation approaches. Transportation Research Part B 41(1), 49-62.

De Jong, D., Knoop, V.L., Hoogendoorn, S.P., 2013, The effect of signal settings on the macroscopic fundamental diagram and its applicability in traffic signal driven perimeter control strategies, IEEE Conference on Intelligent Transportation Systems, Proceedings ITSC, 1010-1015.

Eichler, M., Daganzo, C. F., 2006, Bus lanes with intermittent priority: Strategy formulae and an evaluation, Transportation Research Part B 40(9), 731-744.

Geroliminis, N., Boyaci, B., 2012, The effect of variability of urban systems characteristics in the network capacity, Transportation Research Part B 46, 1607-1623.

Geroliminis, N., Daganzo, C.F., 2008, Existence of urban-scale macroscopic fundamental diagrams: some experimental findings, Transportation Research Part B 42(9), 759770.

Geroliminis, N., Zheng, N., Aboudolas, K., 2014, Document A three-dimensional macroscopic fundamental diagram for mixed bi-modal urban networks, Transportation Research Part C 42, 168-181.

Godfrey, J., 1969, The mechanism of a road network, Traffic Engineering and Control 11(7), 323-327.

Gonzales, E.J., Daganzo, C.F., 2012, Morning commute with competing modes and distributed demand: user equilibrium, system optimum, and pricing, Transportation Research Part B 46(10), 1519-1534.

Haddad, J., Geroliminis, N., 2012, On the stability of traffic perimeter control in tworegion urban cities, Transportation Research Part B 46, 1159-1176. 
584 Haddad, J., Shraiber, A., 2014, Robust perimeter control design for an urban region, Transportation Research Part B 68, 315-332.

Hans, E., Chiabaut, N., Leclercq, L., 2014, Investigating the irregularity of bus routes: highlighting how underlying assumptions of bus models impact the regularity results, Journal of Advanced Transportation, http://dx.doi.org/10.1002/atr.1275.

Hans, E., Chiabaut, N., Leclercq, L., 2014a, A Clustering Approach to Assess the Travel Time Reliability of Arterials, Transportation Research Record: Journal of the Transportation Research Board, 2422, 42-49.

Keyvan-Ekbatani, M., Kouvelas, A., Papamichail, I., Papageorgiou, M., 2012, Exploiting the fundamental diagram of urban networks for feedback-based gating, Transportation Research Part B 46, 1393-1403.

Leclercq, L., Geroliminis, N., 2013, Estimating MFDs in Simple Networks with Route Choice, Transportation Research Part B 57, 468-484.

Leclercq, L., Chiabaut, N., Trinquier, B., 2014, Macroscopic fundamental diagram: a crosscomparison of estimation method, Transportation Research Part B 62, 1-12.

Mahmassani, H.S., Williams, J.C., Herman, R., 1984, Investigation of the network-level traffic flow relationships: some simulation results, Transportation Research Record 971, 121-130.

Wardrop, J.G., 1952, Some theoretical aspects of road traffic research, Institution of Civil Engineers 2, 325-378.

Xie, X., Chiabaut, N., Leclercq, L., 2013, Macroscopic Fundamental Diagram for Urban Streets and Mixed Traffic: Cross-comparison of Estimation Methods. Transportation Research Record 2390, 1-10.

Zheng, N., Geroliminis, N., 2013, On the distribution of urban road space for multimodal congested networks, Transportation Research Part B 57, 326-341. 